\title{
Overview of HADES Results
}

\section{Manuel Lorenz for the Collaboration*}

Institut für Kernphysik, Goethe-Universität, 60438 Frankfurt, Germany

E-mail: Lorenz@Physik.uni-frankfurt.de

\begin{abstract}
HADES is a multi-purpose charged-particle detector operated at the SIS18 synchrotron located at the GSI Helmholtz Center for Heavy Ion Research in Darmstadt, Germany. The provided ion beam energies of 1-2 A GeV are among the lowest of all currently running heavy-ion experiments and result in the highest baryo-chemical potentials at freeze-out in case of Au+Au collisions. The HADES heavy-ion program focuses on various observables: (sub-threshold) strangeness production, particle flow and its anisotropies, virtual photon emission and net-proton number fluctuations and is supplemented by more elementary reactions induced by protons and pions. We compare the modeling of heavy-ion collisions at HADES with neutron star collisions and show that they probe the same part of the phase diagram. We further discuss the different contributions adding up to the finally measured hadron spectra using $\mathrm{K}^{-}$mesons as an example.
\end{abstract}

Critical Point and Onset of Deconfinement - CPOD2017

7-11 August, 2017

The Wang Center, Stony Brook University, Stony Brook, NY

${ }^{*}$ Speaker. 


\section{The Baryon-Rich Side of the Phase Diagram}
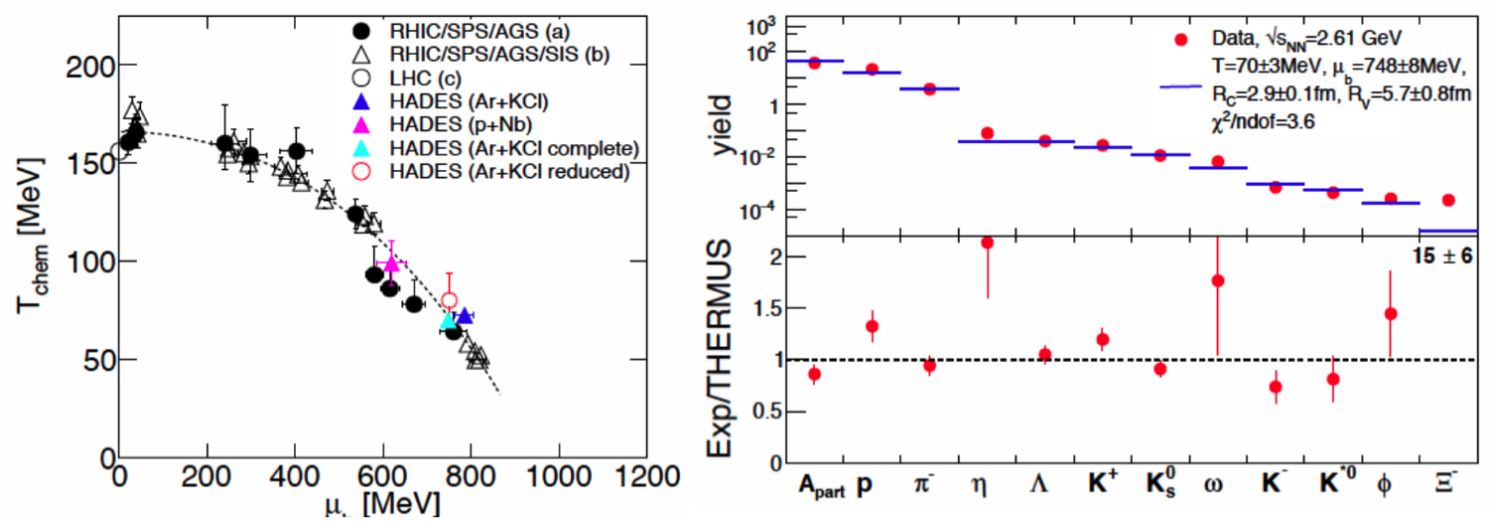

Figure 1: Left panel: Chemical freeze-out points in the $T_{\text {chem }}-\mu_{b}$ plane. The filled black circles (a) are taken from [8], the black open triangles (b) are from [6] and the black open circle (c) from [7]. The presented fit results are defined in the legend. The dashed curve corresponds to a fixed energy per nucleon of $1 \mathrm{GeV}$, calculated according to [6] and various fits to HADES data [9, 10]. Right panel: Yields (filled red circles) of hadrons in $\mathrm{Ar}+\mathrm{KCl}$ reactions and the corresponding THERMUS fit values (blue bars). The lower plot shows the ratio of the experimental value and the THERMUS value. For the $\Xi^{-}$, the value of the ratio is quoted instead of displaying a point.

The characterizations of strongly interacting matter in its different phases is a great challenge. Especially, at high net-baryon densities properties of QCD matter are not well established as abinitio calculations can not yet be performed in this regime [1]. Thus, one has to rely on extrapolations or models based on effective Lagrangians. This lack of exact QCD calculations, opens up for a range of various possible scenarios, like higher order phase transitions and new exotic phases of matter [2], which need to be explored experimentally.

Heavy-ion collisions (HICs) are the tool for this endeavor. As the inter-penetration time of the colliding nuclei decreases with increasing collision energy and the amount of stopped nucleons in the collision zone decreases at the same time, the net-baryon density in the collision zone increases with decreasing energy. On a more quantitative level, the extracted freeze-out parameters from statistical hadronization model (SHM) fits $[3,4,5,8,7]$ to particle yields obtained at various energies show a striking regularity, lining up on a curve in the temperature - baryo-chemical potential plane, connecting smoothly data from the lowest energies at SIS18 up to the highest available energy at LHC [6, 7], see left panel of Fig.1. An example for such a fit to HADES data from Ar+KCl collisions at $\sqrt{s_{N N}}=2.6 \mathrm{GeV}[9,10]$, obtained with the THERMUS code [11], is depicted on the right panel of Fig.1. Thus, HICs offer a unique possibility for a systematic scan of the different phases of strongly interacting matter in the laboratory.

HADES is a multi-purpose charged-particle detector operated at the SIS18 synchrotron located at the GSI Helmholtz Center for Heavy Ion Research in Darmstadt, Germany. The provided ion-beam energies of 1-2 A GeV translate to the highest baryo-chemical potentials at freeze-out [6] of all currently running heavy-ion experiments, hence it marks the high baryon density frontier. 


\section{Apparatus and Physics Program}

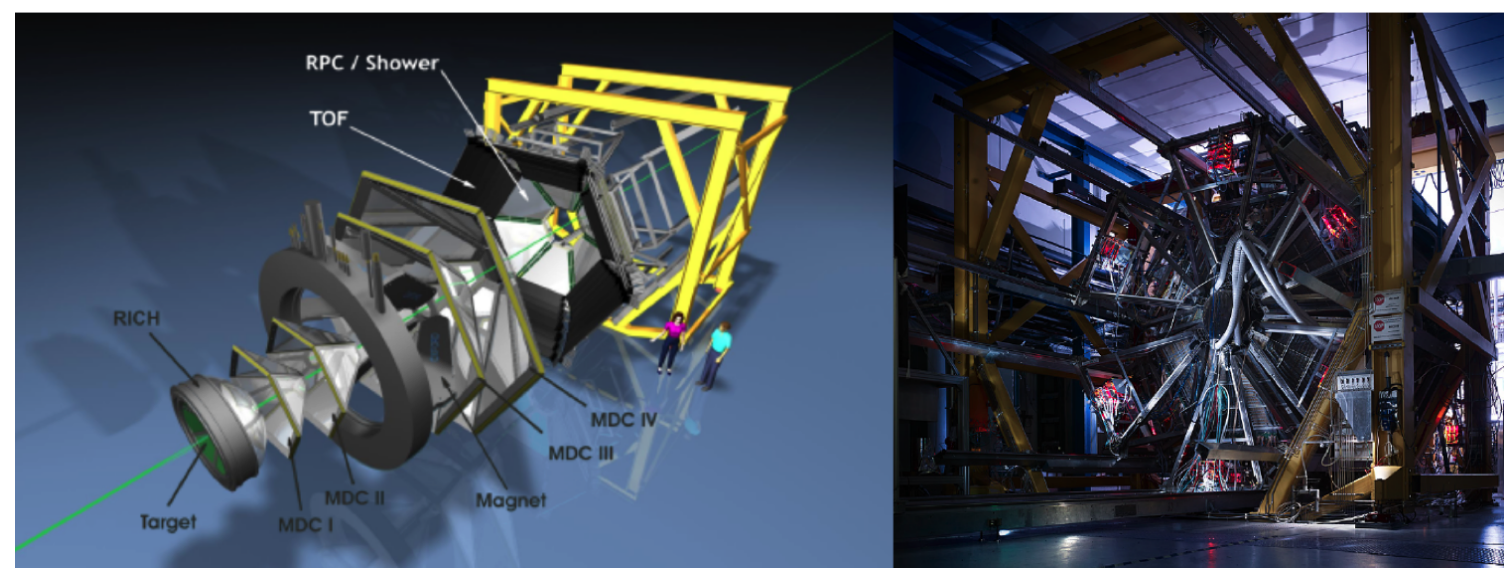

Figure 2: Left panel: Exploded-view of the HADES detector system [13]. Right panel: Photo taken from the back of the apparatus [14].

HADES comprises a 6-coil toroidal magnet centered around the beam axis and six identical detection sections located between the coils, covering almost the full azimuthal angle. Each sector is equipped with a Ring-Imaging Cherenkov (RICH) detector followed by low-mass Mini-Drift Chambers (MDCs), two in front of and two behind the magnetic field, as well as a scintillator hodoscope (TOF) and a resistive plate chamber (RPC) at the end of the system. The RICH detector is used mainly for electron/positron identification, the MDCs are the main tracking detectors, while the TOF and RPC are used for time-of-flight measurements in combination with a diamond start detector located in front of a 15 -folded segmented target. The setup is completed by a forward hodoscope used for event plane determination. A detailed description of the HADES detector is given in [12].

The HADES HIC program focuses on four observables: (sub-threshold) strangeness production (see Sec.3), particle flow and its anisotropies [15], virtual photon emission [16] and net-proton number fluctuations. The physics program is complemented by investigations on more elementary collisions systems using pion and proton induced reactions on nuclei $[17,18]$.

\section{Neutron Star Mergers and HICs at HADES}

The discovery of a gravitational wave signals by the Laser Interferometer Gravitational-Wave Observatory (LIGO) [19] marks the start of the gravitational-wave astrophysics era in which the signal transmitted by the waves is used to probe compact stellar objects in a completely new way offering unique opportunities. Gravitational wave signals are expected to deliver tight constraints on the equation of state of matter [20].

For the modeling of macroscopic collisions of neutron stars one has to solve Einstein equations together with those of relativistic hydrodynamics and magnetohydrodynamics. This needs sophisticated and computing intensive numerical simulations in three dimensions. Nevertheless, a new generation of general-relativistic hydrodynamics codes have been developed, which are able to 

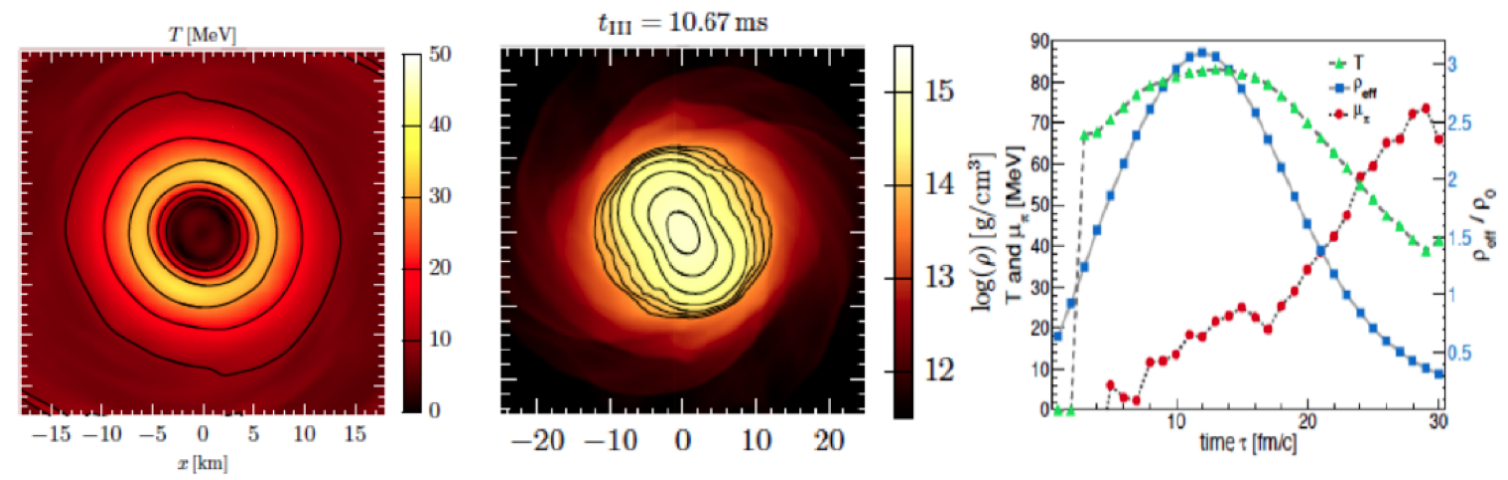

Figure 3: Left panel: Temperature profile in $\mathrm{MeV}$ for a neutron star collision. Middle panel:Density profile of a neutron star collision. Note that nuclear ground state density corresponds to $2 \times 10^{14} \mathrm{~g} / \mathrm{cm}^{3}$. Right panel: Density and temperature evolution for microscopic Au+Au collisions $\sqrt{s_{N N}}=2.4 \mathrm{GeV}$.

describe the evolution of the binary system with a very high convergence rate [21]. Full scale simulations of the evolution of the binary system show temperatures of up to $50 \mathrm{MeV}$ (left panel of Fig. 3) and densities of 2-4 times nuclear ground state density (panel of Fig. 3) for the inner part of the collision [22].

With the help of kinetic transport theory, also the dynamical evolution of the microscopic collision of heavy-ions can be modeled. The density and temperature evolution for $\mathrm{Au}+\mathrm{Au}$ collisions at $\sqrt{s_{N N}}=2.4 \mathrm{GeV}$ as measured by HADES extracted from the transport model UrQMD [23, 24] is displayed on the right panel of Fig 3. It is visible that the macroscopic as well as the microscopic collision system probe indeed very similar regions of the phase diagram of strongly interacting matter, hence the establishment of a connection of both systems is a logical step and promises a unique interplay.

The tight constraints for the equation of state by neutron star mergers might be the standard candle at high baryon densities needed to reduce the degrees of freedom in modeling of HIC such that one can seriously attack the fundamental process of hadronization and the formation of matter, we have been prevented of gaining a deeper understanding in the last 40 years. Yet, due to the very different time-scales of the two types of collisions, a remaining uncertainty is the role of strangeness production through the weak interaction, which might be essential for the stability of neutron stars [25].

\section{Unique Observable: Sub-threshold Strangeness Production}

Newly produced quarks deliver important information on the created fireball. In addition, at energies below $\sqrt{s_{N N}} \approx 2.55 \mathrm{GeV}$, strange quarks can not be produced in binary nucleon-nucleon collisions because of the higher production threshold of the lightest hadrons carrying strangeness. However, in heavy-ion reactions additional multi-step processes do occur and a production of hadrons below their free $\mathrm{N}+\mathrm{N}$ threshold is possible by accumulation of energy. Therefore, the investigation of sub-threshold strangeness production is one of the most promising probes, as they contain newly produced quarks, are sensitive to the amount of energy provided from the created 


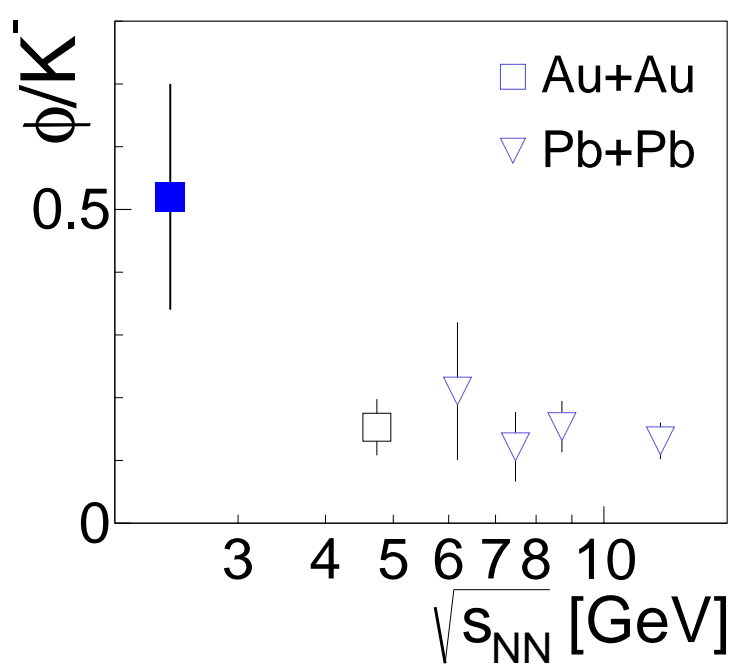

Figure 4: Multiplicity ratio $\phi / K^{-}$as function of $\sqrt{s_{N N}}[28,29]$ for central heavy-ion collisions. The HADES data are depicted as filled symbol.

system and the equation of state.

Recently, HADES has been able to show that it is very important to measure all relevant hadron states carrying strangeness in order to achieve a complete picture of the production and propagation of strangeness inside the fireball.

The first simultaneous measurement of $K^{-}$and $\phi$ mesons in central Au+Au collisions at $\sqrt{s_{N N}}=$ $2.4 \mathrm{GeV}$ by HADES revealed that the $\phi / K^{-}$multiplicity ratio is found to be surprisingly high with a value of $0.52 \pm 0.16$, see Fig. 3. Consequently, the different slopes of the $K^{+}$and $K^{-}$transversemass spectra can be explained solely by feed-down, which substantially softens the spectra of $K^{-}$ mesons [26, 27].

Here, we extend the analysis to the yet not reconstructed $\Lambda(1520)$ in this collision system. Although the $\Lambda(1520)$ multiplicity is expected to be small, due to its large branching ratio to a final state involving a $K^{-}$of 0.23 , it might turn out to give a relevant contribution to $K^{-}$production. We use the THERMUS code, which has been shown before to reproduce hadron yields with high accuracy, to estimate the ratio $\Lambda(1520) / K^{-}$at freeze-out to be 0.74 . Taking the branching ratio into account, this translates to $16 \%$ of all observed $K^{-}$resulting from $\Lambda(1520)$ decays.

As in our previous work, we investigate the effect of the feed-down contribution on the finally observed $K^{-}$transverse mass spectra by a convolution of thermal sources generate with the event generator Pluto [30]. According to the measured $\phi / K^{-}$multiplicity ratio, 26\% of $K^{-}$mesons result from $\phi$ decays. The $\phi$ mesons are generated from a thermal source characterized by the measured inverse slope of the $\phi$ mesons of $\mathrm{T}=108 \mathrm{MeV}$. For the $\Lambda(1520)$ source, from which $16 \%$ of the $K^{-}$ are emitted, we use $\mathrm{T}=100 \mathrm{MeV}$, which is in accordance with the measured preliminary inverse slope of the $\Lambda$ hyperon [31]. The remaining 58\% of $K^{-}$mesons come directly, from a thermal source with a temperature of $\mathrm{T}=104 \mathrm{MeV}$ according to the measured inverse slope of the $K^{+}$. The various contributions to the transverse mass spectra of $K^{-}$mesons around mid-rapidity, as well as the sum (cocktail) of the different contributions are compared to experimental data in Fig. 4. The 
resulting sum (cocktail) of the three contributions is then fitted using the following equation:

$$
\frac{1}{m_{t}^{2}} \frac{d^{2} M}{d m_{t} d y_{c m}}=C\left(y_{c m}\right) \exp \left(-\frac{\left(m_{t}-m_{0}\right)}{T_{B}\left(y_{c m}\right)}\right)
$$

The extracted slope of $81 \mathrm{MeV}$ agrees with the measured slope of $K^{-}$of $(84 \pm 6) \mathrm{MeV}$. Hence, it becomes clear that it is misleading to interpret the measured inverse slope of hadron spectra as a kinetic freeze-out temperature as it is a convolution of various sources with additional effects, e.g. radial expansion of the fireball on top. Hence, the different slopes of charged kaons do not imply diverging freeze-out temperatures of these two mesons caused by unequal coupling to baryons, as suggested in past literature [27]. Note, that due to the hierarchy in production yields, the feed-down is negligible on the $K^{+}$spectra.

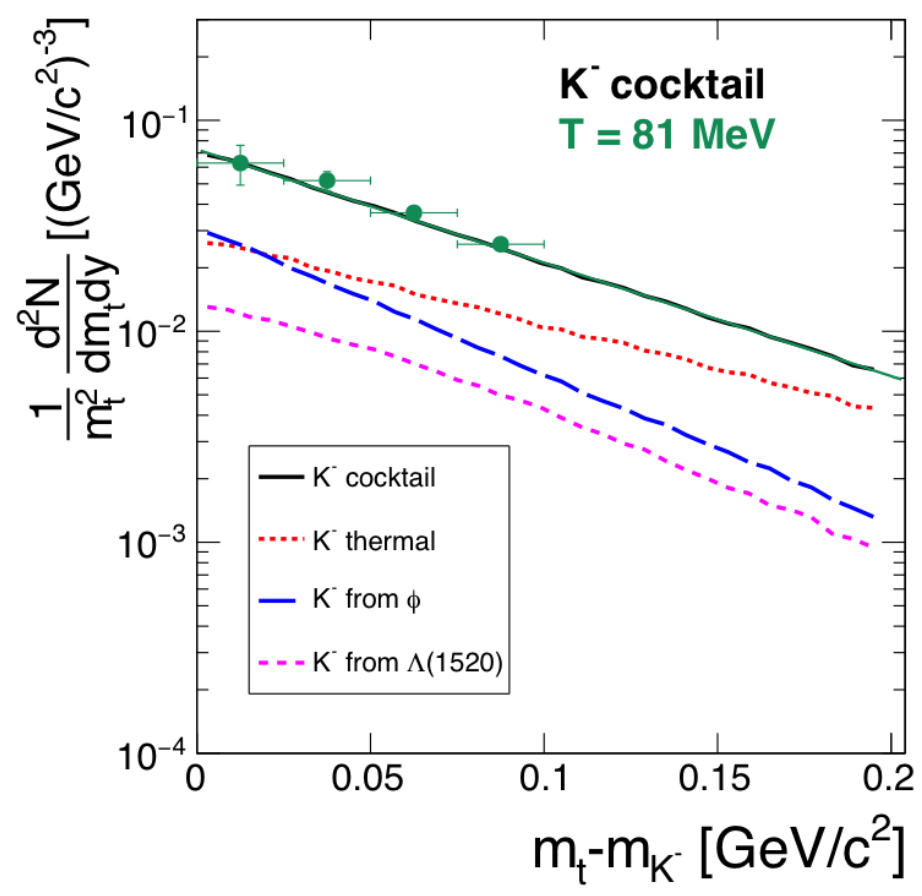

Figure 5: Various contributions to the transverse mass spectra of $K^{-}$mesons around mid-rapidity compared to experimental data from [27] and a Boltzman fit to the sum (cocktail) of the different contributions (green line). The extracted slope of $81 \mathrm{MeV}$ agrees with the measured slope of $K^{-}$of $(84 \pm 6) \mathrm{MeV}$.

\section{Summary}

HADES is a multi-purpose charged-particle detector focusing on various observables in heavyion collisions such as (sub-threshold) strangeness production, particle flow and its anisotropies, virtual photon emission and net-proton number fluctuations. The heavy-ion program is supplemented by more elementary reactions induced by protons and pions. HICs at HADES mark the 
high baryo-chemical potential frontier and probe according to models the same region of the phase diagram as neutron star mergers. We further showed that various different contributions are adding up to the measured hadron spectra of $K^{-}$mesons, hence the inverse slope of the spectra should not be directly interpreted as a temperature and the difference in slopes of charged kaons do not imply diverging freeze-out temperatures caused by unequal coupling to baryons of these two mesons, as suggested in literature in the past.

Acknowledgment: We thank Che-Ming Ko for pointing us to the sizable contribution from the $\Lambda(1520)$ to the $K^{-}$yield and gratefully acknowledge the support by the grants SIP JUC Cracow (Poland), 2013/10/M/ST2/00042; TU Darmstadt (Germany), VH-NG-823; GU Frankfurt, (Germany), BMBF:05P15RFFCA, HIC for FAIR, ExtreMe Matter Institute EMMI; TU München, Garching (Germany), MLL München, DFG EClust 153, DFG FAB898/2-1, BmBF 05P15WOFCA; JLU Giessen (Germany), BMBF:05P12RGGHM; IPN, IN2P3/CNRS (France); NPI CAS Rez (Czech Republic), GACR 13-06759S, MSMT LM2015049.

\section{References}

[1] O. Philipsen, EPJ Web Conf. 13703016 (2017).

[2] A. Andronic, D. Blaschke, P. Braun-Munzinger, J. Cleymans, K. Fukushima, L. D. McLerran, H. Oeschler and R. D. Pisarski et al., Nucl. Phys. A 837 (2010) 65.

[3] P. Braun-Munzinger, K. Redlich and J. Stachel, arXiv:nucl-th/0304013 (2003).

[4] A. N. Tawfik, Int. J. Mod. Phys. A 29 17, 1430021 (2014).

[5] M. Floris, Nucl. Phys. A 931103 (2014).

[6] J. Cleymans, H. Oeschler, K. Redlich and S. Wheaton, Phys. Rev. C 73, 034905 (2006).

[7] J. Stachel, A. Andronic, P. Braun-Munzinger andK. Redlich, J. Phys. Conf. Ser. 509, 012019 (2014).

[8] A. Andronic, P. Braun-Munzinger and J. Stachel, Nucl. Phys. A 772, 167 (2006).

[9] G. Agakishiev et al. [HADES Collaboration], Eur. Phys. J. A 47 (2011) 21.

[10] G. Agakishiev et al. [HADES Collaboration], Eur. Phys. J. A 52 (2016) no.6, 178.

[11] S. Wheaton and J. Cleymans, Comput. Phys. Commun. 180, 84 (2009).

[12] G. Agakishiev et al. [HADES Collaboration], Eur. Phys. J. A 41, (2009) 243.

[13] Visualization Dr. Alexander Schmah.

[14] Jan Michael Hosan - HA Hessen Agentur GmbH.

[15] B. Kardan [HADES Collaboration], Nucl. Phys. A 967 (2017) 812.

[16] T. Galatyuk [HADES Collaboration], Nucl. Phys. A 967 (2017) 680.

[17] B. Ramstein [HADES Collaboration], AIP Conf. Proc. 1735 (2016) 080001.

[18] P. Salabura et al. [HADES Collaboration], Nucl. Phys. News 25 (2015) no.2, 22.

[19] B. P. Abbott et al. [LIGO Scientific and Virgo Collaborations], Phys. Rev. Lett. 116 (2016) no.6, 061102 . 
[20] J. S. Read, C. Markakis, M. Shibata, K. Uryu, J. D. E. Creighton and J. L. Friedman, Phys. Rev. D 79 (2009) 124033.

[21] L. Baiotti and L. Rezzolla, Rept. Prog. Phys. 80 (2017) no.9, 096901.

[22] M. Hanauske, K. Takami, L. Bovard, L. Rezzolla, J. A. Font, F. Galeazzi and H. Stoecker, Phys. Rev. D 96 (2017) no.4, 043004.

[23] S. A. Bass et al., Prog. Part. Nucl. Phys. 41 (1998) 225.

[24] T. Galatyuk, P. M. Hohler, R. Rapp, F. Seck and J. Stroth, Eur. Phys. J. A 52 (2016) no.5, 131.

[25] J. Schaffner-Bielich, Nucl. Phys. A 804 (2008) 309.

[26] M. Lorenz et al. [HADES Collaboration], PoS (BORMIO2010) (2010) 038.

[27] J. Adamczewski-Musch et al. [HADES Collaboration], arXiv:1703.08418 [nucl-ex].

[28] B. Holzman et al. (E917), Nucl. Phys. A 698 (2002) 643.

[29] S. V. Afanasiev et al. (NA49), Phys. Lett. B 491 (2000) 59.

[30] I. Fröhlich et al., J.Phys. Conf. Ser. 219 (2010) 032089.

[31] T. Scheib, PhD Thesis Goethe-University Frankfurt, (2017). 